\title{
3D Carbon-based scaffolds for brain models and tissue engineering
}

\author{
Belén CORTÉS-LLANOS ${ }^{1, *}$, Francesco Paolo ULLOA SEVERINO ${ }^{2,3, *}$ \\ ${ }^{1}$ Department of Bioengineering, University of Washington, 3720 15th Ave NE, Seattle, WA 98105, USA. \\ Cell Biology Department, 335 Nanaline Duke Building, Duke University Medical Center, Durham, NC 27710, USA. \\ ${ }^{3}$ Rigeneration Next Initiative, Duke University, Durham, North Carolina, USA. \\ *Correspondence: bcortes@uw.edu; francesco.ulloa@duke.edu \\ https://doi.org/10.37175/stemedicine.v1i4.61
}

\begin{abstract}
Tissue regeneration is probably the most ambitious aim for the tissue engineering research field. Even more difficult it becomes when we attempt to regenerate a complex organ that we do not fully understand, such as the brain. That is why in recent years we have observed an increased number of approaches that strive to create functional brain or networks in vitro in order to study their properties and develop platforms that can be used for biomedical applications. In this review, we will describe how carbon-based materials took over all the other materials as the most interesting and promising platform not only in the electronic industry but also to create 3D functional models of the brain in vitro.
\end{abstract}

Keywords: Carbon-based material · Graphene - Three-dimensional scaffolds · 3D brain models · Tissue engineering platforms

\section{Introduction}

Understanding the interaction between nanomaterials and biosystems has become crucial in recent years. This growing interest has generated novel nanostructures such as nanoparticles (1), nanowires (2), nanotubes (3) and nanofibers (4) that can be applied for biomedical applications. In neuroscience, for example, magnetic or semiconductors materials have been used to study cell migration, particle internalization and electrophysiological properties of neurons (5-8). Conductive polymers, on the other hand, could have not only the electrical properties of metals or semiconductors but also the suitable mechanical properties (i.e. low stiffness) that are necessary to improve neural attachment and growth (9). Recently, new material-based models that can mimic the central nervous system (CNS) have been produced and described. Their physical, mechanical, chemical and electrical properties are carefully characterized to

Received: Jun 17, 2020; Accepted: Aug 31, 2020

(c) The Author(s). 2020 This is an Open Access article distributed under the terms of the Creative Commons License (http://creativecommons.org/licenses/by/4.0/) which permits unrestricted use, distribution, and reproduction in any medium or format, provided the original work is properly cited. understand if they are suitable to be applied as successful platforms for applications in neuroscience $(10,11)$. Among all, carbon-based materials (CBMs) are considered one of the most interesting to study and apply in biomedicine (12). When used alone, in combination with other CBMs or by making hybrids with natural or synthetic polymers, they have been demonstrated to promote electrical and mechanical interactions with the nervous system. Finally, carbon-based three-dimensional (3D) scaffolds are stateof-the-art in the tissue engineering field, having not only the outstanding conductive properties but also a third dimension that allows to better mimic the natural environment of the cells in vivo. In this review, we will describe these findings to convince the reader that these technologies can be further expanded to develop new in vitro models for the nervous system and new platforms for tissue engineering.

Graphene 3D scaffolds to model the brain in vitro Since 40 years ago, when the first attempt of a 3D culture system was published demonstrating the possibility to maintain the cellular differentiation and organization by using a floating collagen gel (13), an overwhelming number of biomaterials have been discovered or synthesized to recapitulate the "closer to in vivo" behavior 
of cells in vitro. CBMs have emerged from the herd since the discovery of the fullerenes, and now with the discovery of graphene, their applications in material science, medicine and biology have increased even more.

Graphene is theoretically known since 1960 as a twodimensional (2D) single-layer sheet of $\mathrm{sp}^{2}$ hybridized carbon atoms organized in a hexagonal (honeycomb) arrangement. However, it was obtained only in 2004 from Andre Geim and Kostya Novoselov (14). This was the first 2D atomic crystal available to humankind and for their discovery, Geim and Novoselov were awarded the Nobel Prize in Physics in 2010. There are many reasons why graphene is such an attractive material. First of all, graphene has incredible mechanical and physical properties (with a stiffness of $150,000,000$ psi and thermal conductivity of around $5000 \mathrm{~W} / \mathrm{mK}$ ). Then it has high electrical conductivity (with a carrier mobility of $15,000 \mathrm{~cm}^{2} \mathrm{~V}^{-1} \mathrm{~s}^{-1}$ and a resistance of $10 \mathrm{ohms}$ ) and the possibility to be chemically functionalized (15-17). This is why graphene is in the limelight not only for industrial applications but also for a breakthrough in the biomedical, tissue engineering and neuroscience fields.

The biocompatibility of graphene materials with brain cells was shown by N.Li et al. in 2011. They showed that mouse primary hippocampal neurons were able to be kept in culture over a graphene film support allowing neurites sprouting and outgrowth (18). Graphene-based materials were shown to be inert to neurons that were able to preserve their physiological activity, as well as to adhere and grow without any coating with adhesion molecules (19). Graphene films were able to enhance the activity of neural stem cell (NSC)-derived neuronal network (20), and a more recent paper reported the unknown ability of graphene to regulate the extracellular ion distribution, by trapping ions that control the neuronal excitability, and in turn, affecting the neural network activity (21).

How can we move from these $2 D$ graphene-based systems to a $3 D$ graphene-based scaffold to better model and study the brain?

Chemical vapor deposition (CVD) is one of the methods to obtain graphene (17), which allows the synthesis of a 3D graphene-based scaffold using a nickel template that is then removed by etching. CVD was used, for instance, to create an oriented 3D structure of graphene bricks. This novel graphene structure can be customized based on the application by adjusting the pore size, from 10 to $50 \mu \mathrm{m}$, and the angle of the bricks across layers using $45^{\circ}$ or $90^{\circ}$ (22). They have an electrical conductivity value of $60-80$ $\mathrm{Scm}^{-1}$ and a density of $3.6 \mathrm{mgcm}^{-3}$ showing properties as a supercapacitor electrode and flexible conductor. Another 3D scaffold was made by using a nickel foam as a template. This resulted in a hollow, tubular structure made of graphene that is interconnected in three dimensions called 3D graphene foams (3D-GFs) (23) (Figure 1A, left). Ning Li and collaborators used adult NSCs derived from the mouse hippocampus and conducted proof-of-concept studies on the application of $3 \mathrm{D}-\mathrm{GFs}$ as a conductive substrate for cell electrical stimulation. They demonstrated not only the ability of these cells to adhere and grow within the scaffold but also their capability to differentiate into functional neurons, astrocytes and oligodendrocytes that could be electrically stimulated directly passing a current through the 3D-GFs (24). The same group reported, in a paper led by Qing Song, a reduced inflammatory response of NSC-derived microglia growth on the 3D-GFs upon insult with lipopolysaccharide (LPS) (25) demonstrating the anti-inflammatory effect of this cellmaterial interaction. Ulloa Severino and collaborators for the first time were able to culture primary rat hippocampal cells on the 3D-GFs and demonstrated that, using calcium imaging techniques and theoretical models, in 3D there are different neural networks properties (26). They reported that a small-world network model with frustrated synchrony could recapitulate the activity of the neuronal network growth on 3D-GFs that showed local and global network activity, as well as the existence of neuronal assemblies with a correlated activity that varies in space and time. These phenomena were similar to the one found in vivo making the $3 \mathrm{D}-\mathrm{GF}$ hippocampal cultures a better in vitro system to study the brain. They showed that these results were not only due to the interactions between brain cells and graphene but also to the development of many more processes and connections along the third dimension, as well as to morphological changes in the shape of astrocytes (26), another important component of brain networks. The limitation of this scaffold was that the cells, although sometimes crossing the pores of the GF (Figure 1A, right), were adhering and growing on a 2D surface developed in 3D. How can these scaffolds be improved to have a real 3D culture system? What are the proper modifications and improvements that need to be made?

\section{Hybrid carbon-based scaffolds for $3 D$ culture systems}

Graphene is obviously not the only CBM used for biomedical applications in neurology (12). Indeed, the most studied CBM before graphene was carbon nanotubes (CNTs). CNTs are cylindrical hallow graphene structures that can exist in the form of single-wall CNT (SWCNT) or multi-wall CNT (MWCNT). As can be inferred by the names, the difference is in the number of graphene sheets which form the tubular shape and in turn determine the diameter (from approximately $1 \mathrm{~nm}$ for SWCNTs to 100 nm for MWCNTs) (27). CNTs present good thermal and chemical stability as well as high mechanical strength. Moreover, thanks to their electron-rich properties, CNTs are well suited to interface electrically active tissues such as the heart and the brain. For biological applications, different substrates have been used to deposit 2D CNTbased bricks such as forests of vertically aligned CNTs (28), films of CNT building blocks (29) as well as extended 2D meshes of CNT (30), but the limitation was that cells could not migrate into the deep layers of these CNT assemblies. However, interfacing CNTs with brain tissues was reported to boost the electrical activity, sustain the survival of neurons, modulate neuronal growth as well as promote the functional reconnection of segregated 
A

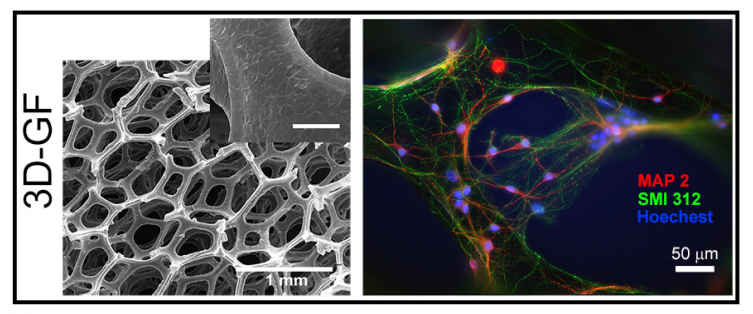

B

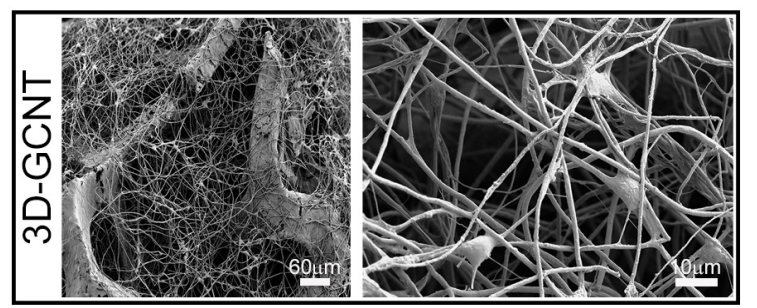

Figure 1. (A) An example of 3D-GF, on the left a scanning electron microscopic image showing the big pore size, and on the right mature neurons growing on the backbone and sometimes able to cross the pore of the structure without support. (B) An example of 3D-GCNT, on the left showing the CNT web inside the GF structure, and on the right showing the intricate web of CNT supporting the neuronal cell bodies. Modified from Ulloa Severino et al., 2016 (26).

spinal cord slices (30-33). Aiming to improve the in vitro model for the application of CNTs in neuroscience, Bosi et al. fabricated a polydimethylsiloxane (PDMS) 3D porous structure with the inner walls of the pores layered with MWCNTs. In this way, they were able to maintain the mechanical properties of the PDMS structure with the addition of the electrical and physical properties of CNTs (34). These structures were used to culture primary hippocampal cells in vitro to show that the network activity, in terms of synchronization and frequency, did not change between 2D and 3D MWCNTs but was anyway higher than both 2D and 3D PDMS substrates alone, in which the third dimension made the difference (34). These same scaffolds were then used to show their ability to functionally reconnect segregated spinal cord slices (35). In the same paper, the authors also showed the effect of the scaffold implant in vivo. After 4-8 weeks from the implant in the rat visual cortex, there was no increase in the inflammatory response in the tissue nearby the implant ( $50 \mu \mathrm{m}$ from the implant) and a significant reduction at further distance (up to $150 \mu \mathrm{m}$ from the implant), measured as intensity of glial fibrillary acidic protein (GFAP) and Ionized calcium binding adaptor molecule 1 (Iba1) immunofluorescence as indication of glia reaction after injury. Finally, they demonstrated that neurons were able to infiltrate the scaffold with their processes and soma, demonstrating a good integration of the exogenous material into the tissue as early as 2 weeks post-implant.

The idea of fabricating scaffolds of different materials to integrate their best properties is fascinating and necessary in order to improve the new platforms for brain-material interface and to find new ways of mimicking the brain in vitro. This approach was exploited very well by Xiao and collaborators who applied the CVD method to fabricate a 3D-GF with a 3D mesh of CNTs filling the pores of the GF backbone (Figure 1B, left), combining the advantages of both materials (36). These 3D graphene CNTs scaffolds (3D-GCNTs) were used to culture rat cortical cells in 3D. What they observed was a functional cortical network that was growing through the whole structure (more than $600 \mu \mathrm{m}$ in height) made of 3 times more cells than the one counted on the GF. The unexpected observation was that they found cell bodies suspended into the pores with the neurites anchored on the GCNT structure (Figure 1B, right). This in vitro 3D cortical co-culture presented an activity dynamic similar to the one observed in vivo having an increased frequency and synchronization, even compared to the 3D-GFs, as well as a reduction in synchronization with the maturation of the network. The big step ahead of their approach though was to use this cortical co-culture system as a platform for other studies. They showed that the 3D cortical network can be used to study glioblastoma infiltration in a cell dense in vitro brain model using 3D live-cell imaging as well as to screen drugs (36). What they found was that, comparing the bare GCNTs with the ones seeded with the cortical culture, there was a decrease in the speed of infiltration of the glioblastoma cells in the co-culture system. Moreover, the effect of the drugs used to investigate these processes changes between the two conditions. When blebbistatin, a drug used to slow down the infiltration of cancer cells, was used on glioblastoma cells seeded on the $3 \mathrm{D}$ cortical network, whose velocity decreased to a lower extent compared to the GCNT scaffold alone. This had a big impact not only as a novel approach that could be compared to another 3D tissue model system but also for the indications that the environment in which researchers screen drugs can affect their functionality, and having better in vitro models for these types of experiments is extremely important. Many questions remain to be addressed, but what can we do more? Where is this research field going?

\section{Future approaches for the biomaterial interface}

Graphene-based materials have their own characteristics, amount of layers, composition, chemistry surface, purity, defects and oxygen content. Even if graphene oxide (GO) and reduced graphene oxide (rGO) present lower conductivity than graphene, these materials possess better hydrophilic property and versatility than pristine graphene scaffolds, making them more suitable for some different neuroscience applications. In a study published by Serrano and collaborators, rGO scaffolds were built by ice segregation-induced self-assembly technique (37). This material has exceptional mechanical compression properties in the longitudinal and transversal directions. This 3D GO scaffold could be compatible with the nervous tissue mechanical properties $(0.3-1.0 \mathrm{kPa})(38)$. López-Dolado implanted this $3 \mathrm{D}$ scaffold made of $\mathrm{rGO}$ for the first time in injured rat spinal cord in order to 
A $3 D$ GO/PCL Scaffolds
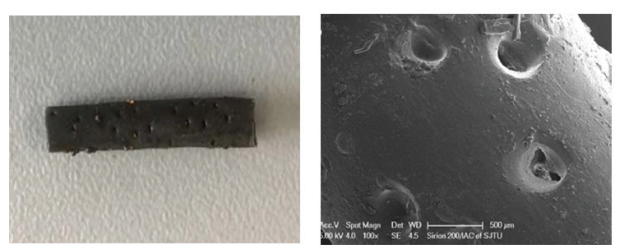

C Regenerated nerves
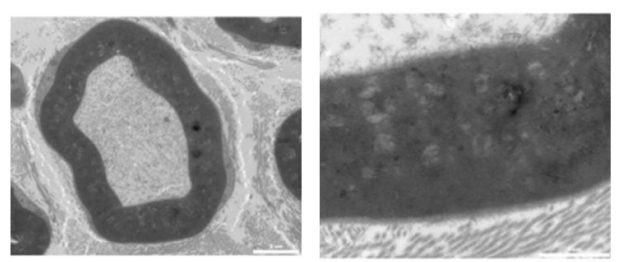

B

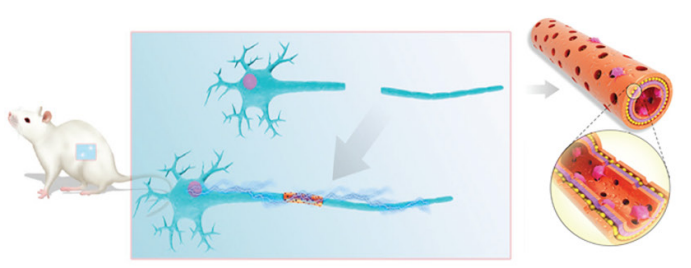

D Angiogenesis

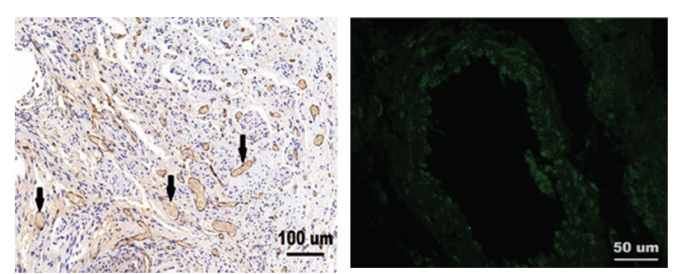

Figure 2. Examples of 3D-GO. (A) Optical (left) and scanning electron microscopic (right) images of GO/PCL scaffold showing the porous structure. (B) Nerve guidance conduits implantation in the rat model and a 3D GO/PCL tubular mold scheme. (C) Transmission electron microscopy images of regenerated nerves (scale left $=2 \mu \mathrm{m}$ and right $=1 \mu \mathrm{m}$ ) in a GO/PCL conduit. (D) Study of angiogenesis in regenerated nerves by immunohistochemistry (left) of endothelial cells (black arrows) and immunofluorescence (right) staining of an associated vascular tissue protein in a GO/PCL scaffold after 18 weeks post injury (scale bars, black $=100 \mu \mathrm{m}$ and white $=50 \mu \mathrm{m}$ ). Modified from Qian et al., 2018 (42).

observe regeneration. After 10 days the scaffold was able to facilitate tissue regeneration. The scaffold prevented the extension of the lesion and established a soft interface at the injury site (39). They also investigated the inflammatory effect of this rGO scaffold after 30 days from implantation by studying vimentin ${ }^{+}$and $\mathrm{ED} 1^{+}$. A reduction in the amount of these two molecules caused a reduction of the inflammatory response. Moreover, rGO scaffolds helped to stabilize and seal the injury as well as support angiogenesis. Inside the structure, they were able to observe blood vessels in the proximity of the regenerated neuronal axons (40). One recent study observed excitatory axons growing in rGO foams through the injury site by functional blood microvessels, implying that these scaffolds improved the neuronal recovery after spinal cord injury (41). These findings demonstrated that rGO could be useful for neuronal regeneration. Qian et al. fabricated GO/polycaprolactone (PCL) nano-scaffolds (Figure 2A) to study the bases of the process of tissue regeneration, as angiogenesis and nerve regeneration by a Sprague Dawley rat model (Figure 2B) (42). Since the scaffolds were fabricated as a multi-layered structure with many pores, the nerve conduit mechanical properties were reinforced. Moreover, the scaffold structure promoted the contact with body fluid as water, oxygen and other nutrients. After 18 weeks of injury GO/PCL repaired a $15 \mathrm{~mm}$ sciatic nerve defect. These scaffolds were able to induce functional and morphological recovery in peripheral nerve regeneration. TEM images showed higher area, diameter, thickness, and the number of regenerated nerves and myelinated axons than other controls (Figure 2C). The microvessel density was higher using GO/PCL scaffolds after 18 weeks of post-injury. The density was evaluated by immunostaining CD31, an endothelial cell involved in angiogenesis, (shown by arrows in Figure 2D left), and CD34, a transmembrane protein associated with vascular tissue. (Figure 2D right).

Among other materials used to fabricate scaffolds, hydrogels are getting attention due to their excellent characteristics as low stiffness, porosity and bioactivity. These properties make these materials suitable to mimic human tissues. With the advent of 3D printing technology, the fabrication of 3D-printed hydrogel-based scaffolds became a common approach to design new scaffolds. However, hydrogels present some limitations regarding their low processability and poor mechanical properties. New research is focusing on combining hydrogels with CBMs in order to improve the biocompatibility, processability, mechanical and electrical properties. Since GO is stable in water suspensions, making this material suitable for a combination with hydrogels, one of the most frequently used 3D printing techniques is direct ink writing. Using this technique, Yao Bin et al. were able to make a 3D-printed graphene aerogel (SF-3D GA) electrode presenting the remarkable properties of CBMs (43). Olate-Moya et al. presented a hybrid nanocomposite hydrogel based on alginate crosslinked with genalin, chondroitin sulfate and GO particles as ink. They used a fourth-generation 3D bioplotter to $3 \mathrm{D}$ print the scaffolds. These 3D-printed hydrogelGO scaffolds presented good biocompatibility and excellent cell proliferation, alignment and distribution along the scaffold (44). They used human adipose tissue-derived mesenchymal stem cells to test these scaffolds, showing that they presented all the outstanding properties to be applied in neuroscience. In another recent study, it was used a different approach to make 3D-printed scaffolds. First, they produced graphene using a method that combined bovine serum albumin with wet ball milling. They used a custom setup where the 
graphene ink was printed on a Kapton polyimide polymer. They grew neuronal cells (rat dopaminergic, N27) and observed that the platform was able to sense electrical signals (45). These pathways open new possibilities of 3D scaffolds for the study and improvement of neuronal brain models.

\section{Safety of 3D carbon-based scaffolds}

3D carbon-based scaffolds are one of the best candidates for the development of functional brain implants. Engineering new scaffolds that can hold the responsibility of helping the regeneration of a damaged nervous system is one major goal of this field. This, however, requires not only to find the most suitable material and the understanding of the cell-material interactions but also to investigate the effects of long-term exposure to these materials and their safety.

The are many routes of exposure to CBMs and we suggest to the reader a thorough review of the effects on human health and on the environment of CBMs published by Fadeel and collaborators (46). Our aim here is to report what is known about the safety of $3 \mathrm{D}$ carbon-based scaffolds, a topic that has not been covered very much so far despite its importance. Many of the studies conducted to assess the biosafety of CBMs in the brain are based on injectable CBMs in suspension, such as few layer graphene, CNTs, fullerene and GO $(46,47)$. This is surely a relevant aspect as we have learned that, for instance, SWCNTs show a higher toxicity compared to MWCNTs when injected in the rodent's brain $(48,49)$. However, is this also the case for $3 \mathrm{D}$ scaffolds made with CNTs or other CBMs?

The idea that this kind of scaffolds may attenuate the inflammatory response comes from an in vitro study conducted by Song and collaborators (25). They evaluated, upon insult with LPS, the production of reactive oxygen species, interleukin-1 $\beta$, tumor necrosis factor- $\alpha$ and nitric oxide by NSC-derived microglia cultured on 2D and 3D graphene substrates. What they found was promising, the $3 \mathrm{D}-\mathrm{GF}$ s could reduce the inflammatory response in the presence of LPS compared to the $2 \mathrm{D}$ cultures. A recent in vivo study reported a reduced inflammatory response when an electro-spun PCL microfiber scaffold coated with self-assembled colloidal graphene was implanted in the striatum or sub-ventricular zone of adult rat brain (50). They observed a reduced infiltration of Iba1+ microglia within the scaffold coated with graphene compared to the uncoated one (bare PCL). In both cases microglia infiltrated only the initial layer of the scaffolds but by the third week, the scaffolds coated with graphene had a reduced distribution and infiltration of microglia compared to the bare PCL. Finally, they reported reduced scar tissue formation around the implant coated with graphene withing 7 weeks from the surgery. Two other studies showed the ability of 3D carbon-based scaffolds to reduce the microglia infiltration and scar tissue formation upon implant, that in turn demonstrated a good integration of the exogenous material within the CNS $(30,35)$. By using a 3D mash of CNTs or a 3D
PDMS + CNT structure implanted into the visual cortex of adult rats, it was demonstrated that both scaffolds were able to reduce the inflammatory response as indicated by the reduced presence, at far distance from the implant edge, of astrocytes and microglia over time (up to 8 weeks after implant) and the finding of neurons within the implants.

Finally, the degradation and toxicity of 3D scaffolds are poorly known. Domínguez-Bajo et al, studied the degradation of 3D rGO scaffolds after 4 months of being implanted in the spinal cord (41). Using TEM they showed how these 3D scaffolds were dissociated and degraded without toxicity. They observed how the thickness of the scaffold wall was changing over time and how pieces of rGO were uptaken. The internalization process and how these uptaken pieces of rGO could terminate under the blood stream are still uncertain. They evaluated the effect of rGO in different organs and no damage or toxicity was found in the kidney, liver, lung or spleen after 4 months of the $3 \mathrm{D}$ scaffold implantation. They associated this result to their lower dose of rGO $\left(250 \mu \mathrm{g}\right.$ per rat, $\left.700 \mu \mathrm{g} \mathrm{kg}^{-1}\right)$ than other studies. More in vivo studies and chemical analysis by using Raman spectroscopy, X-ray photoelectron spectroscopy or nuclear magnetic resonance will be necessary to fully understand the degradation and toxicity at longer time points to assess the safety of implanted 3D CBM scaffolds and apply these technologies in the biomedical field.

\section{Conclusion}

3D CBM scaffolds were presented as a successful model that could mimic the CNS. These scaffolds showed excellent ability to manipulate neuronal activity and presented exceptional properties for neuronal regeneration. From the neurobiological point of view, they were used as a 3D cell culture model of the brain, recapitulating fundamental processes of neural network formation and function. Their applications spanned from studying electrophysiological properties of neurons to promoting the regeneration after spinal cord injury. We have seen them used to study other cellular mechanisms, like cancer cell infiltration and drug screening. Other open applications could be to use them to study how neuronal network activity changes based on the presence of different ratios of excitatory and inhibitory neurons to investigate their relative contribution within neuronal assemblies and generate new $3 \mathrm{D}$ in vitro models of epileptic networks. In regenerative medicine we could functionalize their surface with molecules that can promote cell-material interactions as well as vascularization of the scaffolds to have permanent and integrated implants. These are just a few examples of what can be done, we need to investigate the mechanisms by which they can promote regenerative processes. From the material science perspective, however, there is still work to do regarding the understanding of the biophysical mechanisms underlying the cell-material interactions and how to improve them. When designing these scaffolds, it should also be considered to make them small enough 
in case they will be used as implants in a complex location, but it should also be large enough to be able to manually handle it. It is still necessary to study the longterm effects of their degradation in a living organism and their potential toxicity. These are a few of the many improvements that can be done to these platforms. We believe that the combination of CBMs with hydrogels or biopolymers presents an excellent potential for the development of new state-of-the-art 3D neuronal system scaffolds that will provide answers to these many questions. These approaches will create a new generation of neuronal model systems that will address these challenges, for the improvement and the study of in vitro brain models as well as for the in vivo application and tissue regeneration.

\section{Conflict of interest}

The authors declare that they have no conflict of interest.

\section{References}

1. Ognjanović M, Radović M, Mirković M, Prijović Ž, Puerto Morales M del, Čeh M, et al. 99mTc-, 90Y-, and 177Lulabeled iron oxide nanoflowers designed for potential use in dual magnetic hyperthermia/radionuclide cancer therapy and diagnosis. ACS Appl Mater Interfaces. 2019 Nov 6;11(44):41109-17.

2. Cortés-Llanos B, Serrano A, Muñoz-Noval A, UronesGarrote E, del Campo A, Marco JF, et al. Thermal route for the synthesis of maghemite/hematite core/shell nanowires. J Phys Chem C. 2017 Oct 19;121(41):23158-65.

3. Barrejón M, Rauti R, Ballerini L, Prato M. Chemically crosslinked carbon nanotube films engineered to control neuronal signaling. ACS Nano. 2019 Aug 27;13(8):8879-89.

4. Liu Y, Meng F, Zhou Y, Mugo SM, Zhang Q. Graphene oxide films prepared using gelatin nanofibers as wearable sensors for monitoring cardiovascular health. Adv Mater Technol. 2019;4(11):1900540

5. Guryanov I, Naumenko E, Konnova S, Lagarkova M, Kiselev S, Fakhrullin R. Spatial manipulation of magneticallyresponsive nanoparticle engineered human neuronal progenitor cells. Nanomedicine Nanotechnol Biol Med. 2019 Aug 1;20:102038.

6. Gahl TJ, Kunze A. Force-mediating magnetic nanoparticles to engineer neuronal cell function. Front Neurosci. 2018, 12:299.

7. Lee J-H, Zhang A, You SS, Lieber CM. Spontaneous internalization of cell penetrating peptide-modified nanowires into primary neurons. Nano Lett. 2016 Feb 10;16(2):1509-13.

8. Robinson JT, Jorgolli M, Shalek AK, Yoon M-H, Gertner RS, Park H. Vertical nanowire electrode arrays as a scalable platform for intracellular interfacing to neuronal circuits. Nat Nanotechnol. 2012 Mar;7(3):180-4.

9. Abidian MR, Corey JM, Kipke DR, Martin DC. Conductingpolymer nanotubes improve electrical properties, mechanical adhesion, neural attachment, and neurite outgrowth of neural electrodes. Small Weinh Bergstr Ger. 2010 Feb 5;6(3):421-9.

10. Khaing ZZ, Thomas RC, Geissler SA, Schmidt CE. Advanced biomaterials for repairing the nervous system: what can hydrogels do for the brain? Mater Today. 2014 Sep 1;17(7):332-40.

11. Gumera C, Rauck B, Wang Y. Materials for central nervous system regeneration: bioactive cues. J Mater Chem. 2011 May 3;21(20):7033-51.

12. Rauti R, Musto M, Bosi S, Prato M, Ballerini L. Properties and behavior of carbon nanomaterials when interfacing neuronal cells: How far have we come? Carbon. 2019 Mar 1;143:430-46.

13. Emerman JT, Pitelka DR. Maintenance and induction of morphological differentiation in dissociated mammary epithelium on floating collagen membranes. In Vitro. 1977 May 1;13(5):316-28.

14. Novoselov KS, Geim AK, Morozov SV, Jiang D, Zhang Y, Dubonos SV, et al. Electric field effect in atomically thin carbon films. Science. 2004 Oct 22;306(5696):666-9.

15. Fattahi P, Yang G, Kim G, Abidian MR. A review of organic and inorganic biomaterials for neural interfaces. Adv Mater. 2014 Mar 1;26(12):1846-85.

16. Georgakilas V, Otyepka M, Bourlinos AB, Chandra V, Kim $\mathrm{N}$, Kemp KC, et al. Functionalization of graphene: covalent and non-covalent approaches, derivatives and applications. Chem Rev. 2012 Nov 14;112(11):6156-214.

17. Novoselov KS, Fal'ko VI, Colombo L, Gellert PR, Schwab MG, Kim K. A roadmap for graphene. Nature. 2012 Oct 11;490(7419):192-200.

18. Li N, Zhang X, Song Q, Su R, Zhang Q, Kong T, et al. The promotion of neurite sprouting and outgrowth of mouse hippocampal cells in culture by graphene substrates. Biomaterials. 2011 Dec;32(35):9374-82.

19. Fabbro A, Scaini D, León V, Vázquez E, Cellot G, Privitera G, et al. Graphene-based interfaces do not alter target nerve cells. ACS Nano. 2016 Gennaio;10(1):615-23.

20. Tang M, Song Q, Li N, Jiang Z, Huang R, Cheng G. Enhancement of electrical signaling in neural networks on graphene films. Biomaterials. 2013 Sep;34(27):6402-11.

21. Pampaloni NP, Lottner M, Giugliano M, Matruglio A, D’Amico F, Prato M, et al. Single-layer graphene modulates neuronal communication and augments membrane ion currents. Nat Nanotechnol. 2018 Aug;13(8):755-64.

22. Xiao $M$, Kong $T$, Wang $W$, Song $Q$, Zhang $D$, Ma $Q$, et al. Interconnected graphene networks with uniform geometry for flexible conductors. Adv Funct Mater. 2015 Ottobre;25(39):6165-72.

23. Chen Z, Ren W, Gao L, Liu B, Pei S, Cheng H-M. Threedimensional flexible and conductive interconnected graphene networks grown by chemical vapour deposition. Nat Mater. 2011 Jun;10(6):424-8.

24. Li N, Zhang Q, Gao S, Song Q, Huang R, Wang L, et al. Three-dimensional graphene foam as a biocompatible and conductive scaffold for neural stem cells. Sci Rep. 2013;3:1604

25. Song Q, Jiang Z, Li N, Liu P, Liu L, Tang M, et al. Antiinflammatory effects of three-dimensional graphene foams cultured with microglial cells. Biomaterials. 2014 Aug;35(25):6930-40.

26. Ulloa Severino FP, Ban J, Song Q, Tang M, Bianconi G, Cheng $\mathrm{G}$, et al. The role of dimensionality in neuronal network dynamics. Sci Rep. 2016 Luglio;6:29640.

27. Ajayan PM. Nanotubes from carbon. Chem Rev. 1999 Jul 1;99(7):1787-800.

28. Shin SR, Shin C, Memic A, Shadmehr S, Miscuglio M, Jung $H Y$, et al. Aligned carbon nanotube-based flexible gel substrates for engineering bio-hybrid tissue actuators. Adv Funct Mater. $2015 \mathrm{Jul}$ 20;25(28):4486-95.

29. Ren J, Xu Q, Chen X, Li W, Guo K, Zhao Y, et al. Superaligned carbon nanotubes guide oriented cell growth and promote electrophysiological homogeneity for synthetic cardiac tissues. Adv Mater. 2017;29(44):1702713.

30. Usmani S, Aurand ER, Medelin M, Fabbro A, Scaini D, Laishram J, et al. 3D meshes of carbon nanotubes guide functional reconnection of segregated spinal explants. Sci Adv. 2016 Jul 1;2(7):e1600087.

31. Lovat V, Pantarotto D, Lagostena L, Cacciari B, Grandolfo M, Righi M, et al. Carbon nanotube substrates boost neuronal electrical signaling. Nano Lett. 2005 Jun 1;5(6):1107-10.

32. Mattson MP, Haddon RC, Rao AM. Molecular functionalization 
of carbon nanotubes and use as substrates for neuronal growth. J Mol Neurosci. 2000 Jun 1;14(3):175-82.

33. Malarkey EB, Fisher KA, Bekyarova E, Liu W, Haddon RC, Parpura V. Conductive single-walled carbon nanotube substrates modulate neuronal growth. Nano Lett. 2009 Jan 14;9(1):264-8

34. Bosi S, Rauti R, Laishram J, Turco A, Lonardoni D, Nieus $\mathrm{T}$, et al. From 2D to 3D: novel nanostructured scaffolds to investigate signalling in reconstructed neuronal networks. Sci Rep. 2015 Apr 24;5:9562.

35. Aurand ER, Usmani S, Medelin M, Scaini D, Bosi S, Rossell FB, et al. Nanostructures to Engineer 3D Neural-Interfaces: Directing Axonal Navigation toward Successful Bridging of Spinal Segments. Adv Funct Mater. 2018;28(12):1700550.

36. Xiao M, Li X, Song Q, Zhang Q, Lazzarino M, Cheng G, et al. A fully $3 \mathrm{D}$ interconnected graphene-carbon nanotube web allows the study of glioma infiltration in bioengineered 3D cortex-like networks. Adv Mater. 2018;30(52):1806132.

37. Serrano MC, Patiño J, García-Rama C, Ferrer ML, Fierro JLG, Tamayo A, et al. 3D free-standing porous scaffolds made of graphene oxide as substrates for neural cell growth. J Mater Chem B. 2014 Aug 6;2(34):5698-706.

38. Lu Y-B, Franze K, Seifert G, Steinhäuser C, Kirchhoff F, Wolburg $\mathrm{H}$, et al. Viscoelastic properties of individual glial cells and neurons in the CNS. Proc Natl Acad Sci USA. 2006 Nov 21;103(47):17759-64.

39. López-Dolado E, González-Mayorga A, Portolés MT, Feito MJ, Ferrer ML, Monte F del, et al. Subacute tissue response to $3 \mathrm{D}$ graphene oxide scaffolds implanted in the injured rat spinal cord. Adv Healthc Mater. 2015;4(12):1861-8.

40. López-Dolado E, González-Mayorga A, Gutiérrez MC Serrano MC. Immunomodulatory and angiogenic responses induced by graphene oxide scaffolds in chronic spinal hemisected rats. Biomaterials. 2016 Aug 1;99:72-81.

41. Domínguez-Bajo A, González-Mayorga A, Guerrero CR, Palomares FJ, García R, López-Dolado E, et al. Myelinated axons and functional blood vessels populate mechanically compliant rGO foams in chronic cervical hemisected rats. Biomaterials. 2019 Feb 1;192:461-74.

42. Qian Y, Song J, Zhao X, Chen W, Ouyang Y, Yuan W, et al 3D fabrication with integration molding of a graphene oxide/ polycaprolactone nanoscaffold for neurite regeneration and angiogenesis. Adv Sci. 2018;5(4):1700499.

43. Yao B, Chandrasekaran S, Zhang H, Ma A, Kang J, Zhang $\mathrm{L}$, et al. 3D-printed structure boosts the kinetics and intrinsic capacitance of pseudocapacitive graphene aerogels. Adv Mater. 2020, 32(8):1906652.

44. Olate-Moya F, Arens L, Wilhelm M, Mateos-Timoneda $M A$, Engel E, Palza H. Chondroinductive alginatebased hydrogels having graphene oxide for 3D printed scaffold fabrication. ACS Appl Mater Interfaces. 2020 Jan 29;12(4):4343-57.

45. Niaraki Asli AE, Guo J, Lai PL, Montazami R, Hashemi NN. High-yield production of aqueous graphene for electrohydrodynamic drop-on-demand printing of biocompatible conductive patterns. Biosensors. 2020 Jan;10(1):6.

46. Fadeel B, Bussy C, Merino S, Vázquez E, Flahaut E, Mouchet $F$, et al. Safety assessment of graphene-based materials: focus on human health and the environment. ACS Nano. 2018 Nov 27;12(11):10582-620.

47. Baldrighi M, Trusel M, Tonini R, Giordani S. Carbon nanomaterials interfacing with neurons: an in vivo perspective. Front Neurosci. 2016 Jun 9;10:250.

48. Bardi G, Tognini P, Ciofani G, Raffa V, Costa M, Pizzorusso T. Pluronic-coated carbon nanotubes do not induce degeneration of cortical neurons in vivo and in vitro. Nanomedicine Nanotechnol Biol Med. 2009 Mar 1;5(1):96-104.

49. Dal Bosco L, Weber GEB, Parfitt GM, Paese K, Gonçalves COF, Serodre TM, et al. PEGylated carbon nanotubes impair retrieval of contextual fear memory and alter oxidative stress parameters in the rat hippocampus. Biomed Res Int. 2015; 2015: 104135

50. Zhou K, Motamed S, Thouas GA, Bernard CC, Li D, Parkington $\mathrm{HC}$, et al. Graphene functionalized scaffolds reduce the inflammatory response and supports endogenous neuroblast migration when implanted in the adult brain. PloS One. 2016;11(3):e0151589.

51. Hwang DW, Park JB, Sung D, Park S, Min K-A, Kim KW, et al. 3D graphene-cellulose nanofiber hybrid scaffolds for cortical reconstruction in brain injuries. 2D Mater. 2019 Sep;6(4):045043. 